\title{
Measuring Bus Service Reliability: An Example of Bus Rapid Transit in Changzhou
}

\author{
Yueying Huo, Southeast University/Inner Mongolia University \\ Jinhua Zhao, Massachusetts Institute of Technology \\ Wenquan Li, Southeast University \\ Xiaojian Hu, Southeast University
}

\begin{abstract}
The objective of this paper is to analyze service reliability of bus rapid transit (BRT) taking Changzhou BRT as an example. Headway irregularity, potential waiting time, equivalent waiting time, and reliability buffer time are recommended to measure service reliability of BRT. Temporal and spatial distributions and comparisons are analyzed. Findings are that passengers of Changzhou BRT need to budget, on average, an extra 3-5 minutes beyond their typical journey time for selected origin-destination pairs to ensure on-time arrival at destinations with $95 \%$ probability. Extra time budgeted for bus waiting beyond mean waiting time contributes to more than 80 percent of extra time budgeted for a journey, while only 20 percent is budgeted for in-vehicle travel time. Service reliability is best near a route's origin terminal and gradually deteriorates along the route, then improves when approaching the route's end.
\end{abstract}

\section{Introduction}

Bus rapid transit (BRT) combines the efficiency and reliability of a rail service with the operating flexibility and lower cost of a conventional bus service. It has been implemented throughout Latin America, North America, Europe, Southeast Asia, Australia, China, and now, increasingly, in Africa and India (Deng and Nelson 2011). 
In China, BRT has expanded faster than in any other regions over the last five years, with $320 \mathrm{~km}$ of BRT systems in 13 cities (Fjellstrom 2010). Service reliability of a transit system has significant impacts on its providers as well as existing and potential users (Cham 2006). The objective of this paper is to analyze service reliability of BRT taking Changzhou BRT as an example. Specifically, issues including the amount of time passengers need to wait, on average, and the amount of extra time passengers need to budget beyond typical wait time and journey time, on average. Temporal and spatial distributions of measures and comparisons between measures are examined. Potential wait time, which is proposed to measure service reliability but has not been applied yet, is put into practice in this paper. Some suggestions for improving service reliability of Changzhou BRT are brought forward. The framework for analyzing service reliability of Changzhou BRT includes measures and analysis dimensions that can be applied to other BRT systems.

\section{Literature Review}

Service reliability is defined as "the invariability of service attributes which influence the decision of travelers and transportation providers" (Abkowitz et al. 1978). The ability of transport operators to understand and improve reliability relies on their ability to measure it (Uniman 2009). Service reliability can be measured from multi-perspectives based on multi-levels.

Measures from operators' perspectives mainly include on-time performance (OTP) and headway regularity (Cham 2006, Kittelson \& Associates, Inc. et al. 2003). OTP is the fraction of services with schedule deviation within some thresholds (Ryus 2003). Headway regularity is defined in terms of the fraction of observed headways that are within some absolute or relative deviation from the scheduled headway (Cramer 2008). Furth et al. (2006) consider it a coefficient of variation of headway, which is the standard deviation of headway divided by the mean.

Measures from the passenger perspective include waiting time-related measures such as excess waiting time, potential waiting time, and equivalent waiting time. They also contain journey time-related measures such as excess journey time, reliability buffer time, and excess reliability buffer time.

Excess waiting time represents the extra amount of time a passenger waits, on average, beyond the scheduled waiting time (Furth and Muller 2006). To have a high probability of arrival at their destinations on time, passengers must plan on waiting longer than the mean waiting time. The 95th percentile waiting time is often interpreted as budgeted waiting time, guaranteeing arrival at their destinations on time 
at least 95 percent of trips (Furth et al. 2006). The difference between budgeted and mean waiting time is called potential waiting time, which is spent at the destination of the trip (Furth and Muller 2006). Equivalent waiting time is a weighted sum of mean and potential waiting time that expresses passenger waiting cost in equivalent minutes of waiting time spent at stops (Furth et al. 2006).

Journey time includes access, egress, and interchange time; ticket purchase time; platform wait time; on-train time; and closures. Each component has a scheduled value, which represents the amount of time a passenger should normally expect to take to complete this stage. The difference between the measured and scheduled times is an indication of service performance, expressed as excess journey time (Chan 2007). Journey time reliability also can be generally defined by quantifying the spread of journey time distribution. The measure quantifying the spread of journey time is known as the reliability buffer time. Reliability buffer time represents the extra time passengers need to budget beyond the typical journey time to ensure $95 \%$ probability of arriving at their destination on time. Uniman (2009) developed a new measure called excess reliability buffer time by proposing a methodology classifying performance into incident-related and recurrent conditions, which attempted to explain the causes of unreliability by isolating the effects of incidents.

BRT is defined as "a flexible, rubber-tired form of rapid transit that combines stations, vehicles, services, running ways, and ITS elements into an integrated system with a strong image and identity" (Levinson et al. 2003). Current studies in China mainly focus on the physical design and planning of BRT (Xu 2007; Mo 2007). In addition to passenger-carrying capacity, the integration of BRT with other modes and its implementation effectiveness were also studied. Current literature outside China pays more attention to the impacts of BRT on land development and land values (Perk et al. 2010). There is a growing body of evidence suggesting that BRT systems have a positive impact on land value uplift (Deng and Nelson 2011). Capability to lead bus-based transit-oriented development (TOD), impacts on travel behavior, environment, fuel consumption, construction, operation cost, and ridership were also studied.

\section{Methodology}

\section{BRT in Changzhou, China}

Vehicles of Changzhou's BRT are equipped with a Global Positioning System (GPS), and large-scale, archived Automated Vehicle Location (AVL) data provide the 
opportunity for studying service reliability. This study employed one trunk route of Changzhou's BRT, called B1, as a specific study case. Archived AVL data of Route B1, consisting of nearly 700,000 records from August 17-23, 2009, were used. Route B1 is one of the busiest bus routes in Changzhou. Its length is $24.5 \mathrm{~km}$, there are 26 stops with an average space of $980 \mathrm{~m}$. Headway is $2-5$ minutes during peak hours and 6-10 minutes during off-peak hours. Route B1 runs north and south and traverses downtown Changzhou, and its middle six stops are located in the downtown. Six origin-destination (OD) pairs were selected to study service reliability based on OD pair level. OD pair1 (length $5,880 \mathrm{~m}$ ) connects south and downtown, OD pair2 $(6,860 \mathrm{~m})$ connects north and downtown, and OD pair3 $(3,920 \mathrm{~m})$, pair4 $(3,920 \mathrm{~m})$, pair5 $(3,920 \mathrm{~m})$, and pair6 $(4,900 \mathrm{~m})$ cover the entire route by connecting them together. Only OD pair5 covers downtown. Figure 1 illustrates Route B1 and the selected OD pairs.

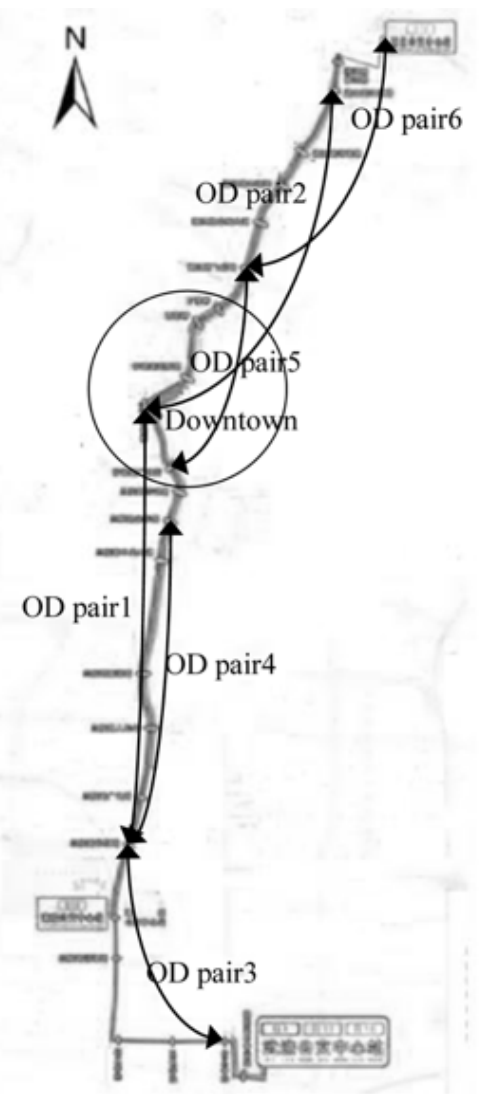

Figure 1. Route B1 and selected OD pairs 


\section{Measures Selection and Calculation}

On-time performance, excess waiting time, excess journey time, headway regularity, potential waiting time (PWT), equivalent waiting time (EWT), and reliability buffer time (RBT) frequently are used for evaluating transit service reliability. On-time performance, excess waiting time, and excess journey time are related to schedule and are suitable for low-frequency services; the other four measures are suitable for high-frequency services. Most BRT systems are high-frequency services; therefore, headway regularity, PWT, EWT, and RBT were selected to measure service reliability of BRT. Headway regularity captures service reliability from the operator perspective based on stop level and is regarded as a coefficient of variation of headway in this paper. The higher the coefficient of variation of headway is, the more irregular the headway is. Headway regularity is called headway irregularity in this paper. PWT and EWT capture service reliability from the passenger perspective based on stop level. RBT captures service reliability from the passenger perspective based on OD pair level. To better understand each measure selected, the definitions, implications, and calculation methods are presented in Table 1.

\section{Table 1. Definitions, Implications, and Calculation Methods for Measures Selected}

\begin{tabular}{|l|l|l|}
\hline \multicolumn{1}{|c|}{ Measures } & \multicolumn{1}{|c|}{ Definitions and Implications } & Calculation Methods \\
\hline $\begin{array}{l}\text { Headway } \\
\text { irregularity }\end{array}$ & $\begin{array}{l}\text { Regarded as coefficient of variation of headway; called } \\
\text { headway regularity in previous studies - the higher it } \\
\text { is, the more irregular the headway is. }\end{array}$ & $\begin{array}{l}\text { Standard deviation of } \\
\text { headway divided by its } \\
\text { mean (Furth et al. 2006) }\end{array}$ \\
\hline $\begin{array}{l}\text { Potential } \\
\text { waiting } \\
\text { time (PWT) }\end{array}$ & $\begin{array}{l}\text { Difference between 95th percentile waiting time and } \\
\text { mean waiting time; extra time that passengers need to } \\
\text { budget beyond mean waiting time for bus waiting to } \\
\text { ensure on-time arrival at destination with 95\% prob- } \\
\text { ability (Furth and Muller 2006). }\end{array}$ & $\begin{array}{l}\text { 95th percentile waiting } \\
\text { time minus mean wait- } \\
\text { ing time }\end{array}$ \\
\hline $\begin{array}{l}\text { Equivalent } \\
\text { waiting } \\
\text { time (EWT) }\end{array}$ & $\begin{array}{l}\text { Weighted sum of mean and potential waiting time that } \\
\text { expresses passenger waiting cost in equivalent minutes } \\
\text { of waiting time spent at stops (Furth et al. 2006). }\end{array}$ & $\begin{array}{l}\text { Mean waiting time + } \\
\text { 0.5 } \times \text { PWT }\end{array}$ \\
\hline $\begin{array}{l}\text { Reliability } \\
\text { buffer time } \\
\text { (RBT) }\end{array}$ & $\begin{array}{l}\text { Difference between 95th percentile journey time and } \\
\text { median journey time; extra time that passengers need } \\
\text { to budget beyond typical journey time for entire jour- } \\
\text { ney to ensure on-time arrival at destination with 95\% } \\
\text { probability (Uniman 2009). }\end{array}$ & $\begin{array}{l}\text { 95th percentile journey } \\
\text { time minus median } \\
\text { journey time (50th per- } \\
\text { centile journey time) }\end{array}$ \\
\hline $\begin{array}{l}\text { Mean } \\
\text { waiting } \\
\text { time }\end{array}$ & $\begin{array}{l}\text { Amount of time between passenger arrival and next } \\
\text { vehicle departure, on average. }\end{array}$ & $\begin{array}{l}\text { 0.5 } \times \text { mean headway } \times \\
(1+\text { ) (Osuna and Newell } \\
1972)\end{array}$ \\
\hline
\end{tabular}


The 95th percentile waiting time, 95 th percentile journey time, and median journey time are needed when calculating PWT and RBT. They can be obtained by interpolation from waiting time distribution and journey time distribution. Waiting time distribution can be estimated based on a set of observed headways according to Equation (1) (Furth and Muller 2006):

$$
F_{W}\left(H_{i}\right)=F_{W}\left(H_{i+1}\right)+i \Delta H_{\mathrm{i}} / \sum_{i=1}^{n} H_{i}
$$

Where,

$W=$ waiting time

$H_{i}=$ ith observed headway; headways numbered in decreasing order

$F_{w}\left(H_{i}\right)=$ waiting time distribution, i.e., probability of $W$ less than or equal to $H_{i} \Delta H_{i}=H_{i}-H_{i+1}$

$n=$ number of observed headways

Journey time for an OD pair is considered the sum of waiting time at the origin stop and the in-vehicle travel time between the origin and destination stops in this paper. Journey time distribution can be estimated based on headways at the origin stop and the in-vehicle travel times of successive trips according to Equation (2) (Ehrlich 2010):

$$
F_{J}(j)=\sum_{i=1}^{n}\left(j-T_{i}\right) / \sum_{i=1}^{n} H_{i}
$$

Where,

$J=$ journey time

$j=$ given time

$T_{i}=$ in-vehicle travel time of the ith trip

$H_{i}=$ headway of ith trip

$F_{j}=$ probability of $J$ less than or equal to $j$

$n=$ number of successive trips. If $j-T_{i}<0$, let $j-T_{i}=0$; if $j-T_{i} \geq H_{i}$, let $j-T_{i}=H_{i}$. 


\section{Analysis Dimensions}

Issues including the amount of time passengers need to wait, the amount of time passengers need to budget beyond typical waiting time and journey time, their variations over time and space, and the relationships between measures can provide comprehensive understanding for service reliability. Therefore, analysis dimensions include value ranges of measures, temporal and spatial distributions of measures, and comparisons between measures. Temporal distribution can be carried on by time period within one day (analyzed here), a weekday and weekend, and a month. Spatial distribution can be carried on by direction, section (analyzed here), and area. The lower the headway irregularity, PWT, EWT, and RBT are, the more reliable the service is.

\section{Results}

\section{Value Ranges of Measures}

Mean headway, headway irregularity, mean waiting time, PWT, and EWT for each stop and RBT for each OD pair were calculated by taking the average number of each hour (06:00 to 21:00), day (August 17-23), and direction (Northbound and Southbound). To explain how the calculations were made, RBT's calculation process of an OD pair is provided below. First, the journey time distribution in each hour was calculated. AVL data were used for the Changzhou BRT for one week and in two directions, so 224 journey time distributions were obtained for each OD pair. Table 2 shows an example of a calculation of journey time distribution. The 95th percentile journey time and median journey time in each hour were gained from the journey time distribution by interpolation, and the RBT in each hour was obtained accordingly. Finally, the RBT of an OD pair was obtained by taking the average number of the 224 values of RBT. 
Table 2. Example of Journey Time Distribution Based on Successive Trips

\begin{tabular}{|c|c|c|c|c|c|c|c|c|c|c|c|c|c|c|c|c|}
\hline \multirow{3}{*}{ Tripi } & \multirow{3}{*}{$\begin{array}{c}\text { Origin Stop } \\
\text { Headway } \\
H_{i}(\text { min) }\end{array}$} & \multirow{3}{*}{$\begin{array}{c}\text { In-Vehicle } \\
\text { Travel Time } \\
T_{i}(\min )\end{array}$} & \multirow{3}{*}{$\begin{array}{c}\text { Max. } \\
\text { Journey Time } \\
H_{i}+T_{i}(\min )\end{array}$} & \multicolumn{13}{|c|}{ Give Time $j$ (min) } \\
\hline & & & & 18 & 19 & 20 & 21 & 22 & 23 & 24 & 25 & 26 & 27 & 28 & 29 & 30 \\
\hline & & & & \multicolumn{13}{|c|}{$j-T_{i}$} \\
\hline Trip1 & 7.15 & 22.80 & 29.95 & 0 & 0 & 0 & 0 & 0 & 0.2 & 1.2 & 2.2 & 3.2 & 4.2 & 5.2 & 6.2 & 7.15 \\
\hline Trip2 & 1.87 & 21.52 & 23.39 & 0 & 0 & 0 & 0 & 0.48 & 1.48 & 1.87 & 1.87 & 1.87 & 1.87 & 1.87 & 1.87 & 1.87 \\
\hline Trip3 & 4.07 & 18.88 & 22.95 & 0 & 0.12 & 1.12 & 2.12 & 3.12 & 4.07 & 4.07 & 4.07 & 4.07 & 4.07 & 4.07 & 4.07 & 4.07 \\
\hline Trip4 & 5.65 & 17.35 & 23.00 & 0.65 & 1.65 & 2.65 & 3.65 & 4.65 & 5.65 & 5.65 & 5.65 & 5.65 & 5.65 & 5.65 & 5.65 & 5.65 \\
\hline Trip5 & 5.75 & 21.70 & 27.45 & 0 & 0 & 0 & 0 & 0.3 & 1.3 & 2.3 & 3.3 & 4.3 & 5.3 & 5.75 & 5.75 & 5.75 \\
\hline Sum & 24.49 & & & 0.65 & 1.77 & 3.77 & 5.77 & 8.55 & 12.7 & 15.08 & 17.08 & 19.08 & 21.08 & 22.53 & 23.53 & 24.49 \\
\hline \multicolumn{4}{|c|}{ Distribution percentile $F_{i}(j)$} & 0.03 & 0.07 & 0.15 & 0.24 & 0.35 & 0.52 & 0.62 & 0.70 & 0.78 & 0.86 & 0.92 & 0.96 & 1 \\
\hline
\end{tabular}


Mean headway was between 3.12-3.96 minutes, which means 15-20 buses are dispatched per hour. Headway irregularity was between $0.34-0.79$. Its span is relatively large, which means headway is irregular from the entire route perspective. Passengers, on average, need to wait 2.17-2.82 minutes and need to budget an extra 3.29-4.54 minutes, on average, for bus waiting to guarantee arrival at their destinations on time with $95 \%$ probability. The extra 3.29-4.54 minutes is spent at destinations. This was transformed into the time spent at stops with a weight of 0.5 . Along with mean waiting time, it was found that passengers need to wait an equivalent of 3.81-5.09 minutes at stops. RBT is between 3-5 minutes, which means passengers need to budget extra 3-5 minutes beyond their typical journey time to ensure $95 \%$ probability of arrival at their destinations on time.

Service Reliability by Time Periods

Figure 2 shows variations of mean headway, headway irregularity, mean waiting time, PWT, EWT, median journey time, and RBT for the Early, AM Peak, Inter-peak, PM Peak, and Evening periods for the Northbound and Southbound directions.

Mean headway is the lowest during $A M$ and PM peaks and highest during the Early period. To be specific, buses are dispatched every 3 minutes during the AM and PM peaks, every 3.7 minutes during Inter-peak, every 4 minutes during Evening, and every 6 minutes during the Early period. Mean headway in the Northbound direction during Evening is higher, at 4.38 minutes.

Mean headway and headway irregularity are highest during the AM and PM peaks, especially during the AM Peak, at above 0.7; they are lowest during the Early period. This means that headways during the AM and PM peaks are less regular than other time periods. This likely is the result of more buses being dispatched during the $A M$ and PM peaks, and traffic conditions during the AM and PM peaks being worse than other time periods.

Mean waiting time is the highest during the Early period, at 3.55 minutes Northbound and 4.05 minutes Southbound. Mean waiting time is also high during the Evening Northbound, close to 3 minutes. This can be attributed to lower service frequencies during these two periods. Mean waiting times during the AM and PM peaks and Inter-peak are below 2.5 minutes. Waiting time in Inter-peak is slightly higher than it is in AM and PM peaks.

Like mean waiting time, PWT and EWT are the highest during the Early and Evening periods Northbound. Relatively low service frequencies during these two periods push passengers to budget more extra time for bus waiting to ensure arrival at their 

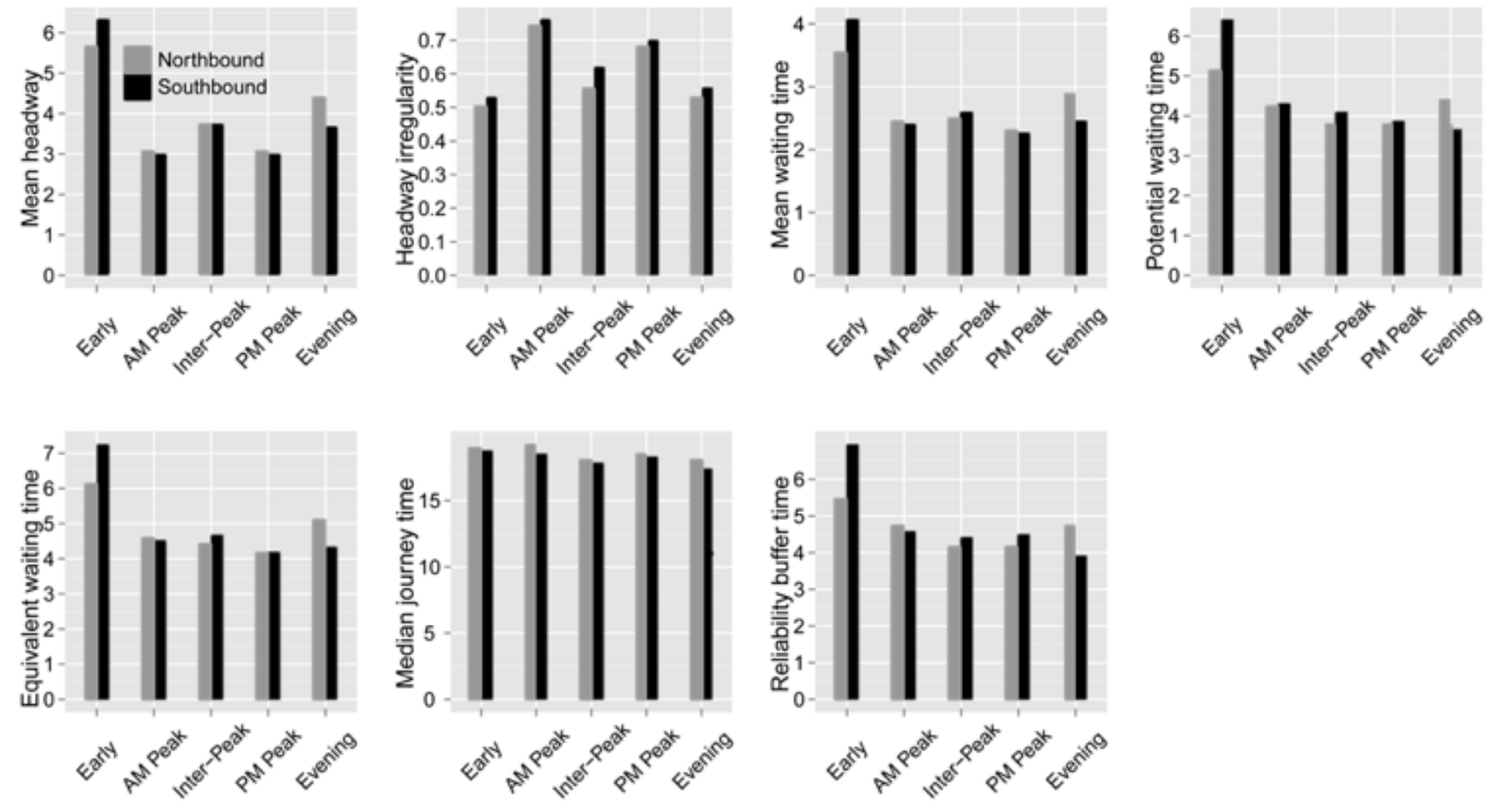

Figure 2. Service reliability by time periods 
destination on time. Higher PWT together with higher mean waiting time during these two periods translates into higher EWT. In other words, equivalent waiting time spent at stops is the highest during the Northbound Early and Evening periods. PWT is slightly higher during AM Peak. This is reasonable because the primary passengers of BRT are office workers and students whose travel purposes during AM Peak are work or study and who want to arrive at their work/study place on time and are willing to budget more time.

The variation of median journey time is small across all time periods. The difference between its maximum, appearing in AM Peak Northbound, and its minimum, appearing in Evening Southbound, is less than 2minutes. Like PWT, RBT is the highest during the Early period, close to 7 minutes Southbound. RBT is also slightly higher during AM Peak, for the same reason as PWT.

In conclusion, service frequency during the Early period is the lowest, which, to a certain extent, results in the highest mean waiting time, PWT, EWT, and RBT during this period. However, headway during the Early period is more regular than any other periods. For the AM and PM peaks, service frequencies are the highest and headway regularity is the worst. Although mean waiting time is the lowest, the difference is negligible compared to other time periods except the Early period. For AM Peak, PWT and RBT are slightly higher than other periods, except for the Early and Evening periods Northbound. From the perspective of operators, i.e., based on headway irregularity, service reliability is the worst during $A M$ and PM peaks. However, from the perspective of passengers based on PWT and RBT, service reliability is the worst during Early period followed by the AM Peak. Relatively worse service reliability during the AM Peak is mainly related to passengers' subjective inclination to budget more time for work or study.

\section{Service Reliability by Directions}

As mentioned above, Route B1 traverses through Changzhou's downtown. Directions are classified into To Downtown (going to downtown from both sides of the route) and From Downtown (leaving downtown from both sides). To analyze mean headway, headway irregularity, mean waiting time, PWT, and EWT by direction, stops in the Downtown were excluded. To analyze median journey time and RBT by direction, OD pair1, OD pair2, OD pair4, and OD pair6 in Figure 1 were selected. Figure 3 shows service reliability by To Downtown and From Downtown. The abscissas of the first five figures in Figure 3 represent stop order regarding the first stop in Northbound as 1. 

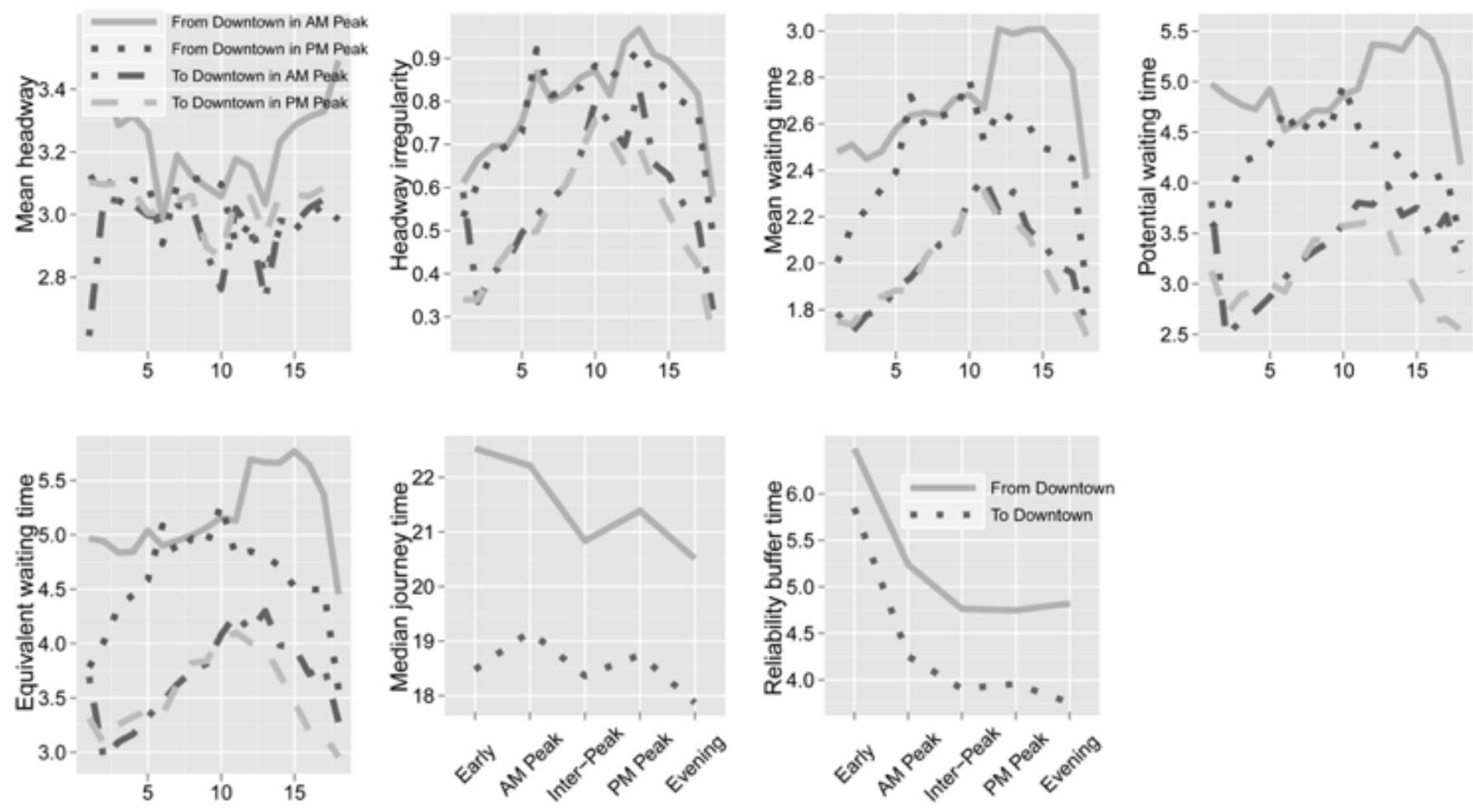

Figure 3. Service reliability by direction 
The common characteristic of headway irregularity, mean waiting time, PWT, EWT, median journey time, and RBT is that they are lower To Downtown than they are From Downtown. Mean headway is lower To Downtown only during the AM Peak. During the AM Peak, higher service frequency To Downtown should be one of the reasons that other measures in this direction are lower. Passengers who travel To Downtown during the $A M$ and PM peaks experience lower mean waiting time, PWT, and EWT, and they also experience lower median journey time and RBT the whole day. Headway is also more regular To Downtown. As a result, service reliability is better To Downtown than it is From Downtown both from the operator and passenger perspectives.

\section{Service Reliability by Sections}

To analyze mean headway, headway irregularity, mean waiting time, PWT, and EWT by section, Route B1 was separated into six sections for Northbound and Southbound, shown as Figure 4. Each section includes $3-5$ stops. Sections are named Section 1 to Section6, with the section closest to the route's origin terminal designated as Section1. Figure 5 shows mean headway, headway irregularity, mean waiting time, PWT, and EWT for Section1-Section 6 for the Northbound and Southbound directions.

Headway irregularity, mean waiting time, PWT, and EWT in Section 1 are the lowest and gradually increase from this section until they reach the maximum in Section 4 and Section5, then begin to decline from Section5. In other words, these measures are lowest at the stops closest to the route's origin terminal, gradually increase as the distance from the route's origin terminal increases, and reaches the maximum at the stops whose distances to the origin terminal account for 80-90 percent of the entire route length. Changzhou BRT sets a schedule for two terminals only, and there is no schedule control for each stop. Buses depart the route's origin terminal according to the schedule, so headway is regular near it. Bus operation is influenced by the external environment, and together with no schedule control en-route, it is difficult to guarantee regular headway en-route near the terminal; the influence gradually cumulates as the distance to the route's origin terminal increases, which translates into increasing headway irregularity, mean waiting time, PWT, and EWT en-route. Drivers want to comply with the schedule when approaching the route's end, so the headway becomes regular again, which results in decreasing headway irregularity, mean waiting time, PWT, and EWT. Mean headway does not show the same variation trend as the other four measures; it is almost the same from Section 1 to Section6, especially Northbound. From a temporal perspective, mean headway shows a difference from the Early to the Evening periods, as shown in Figure 2, which means mean headway is sensitive to time rather than space. 


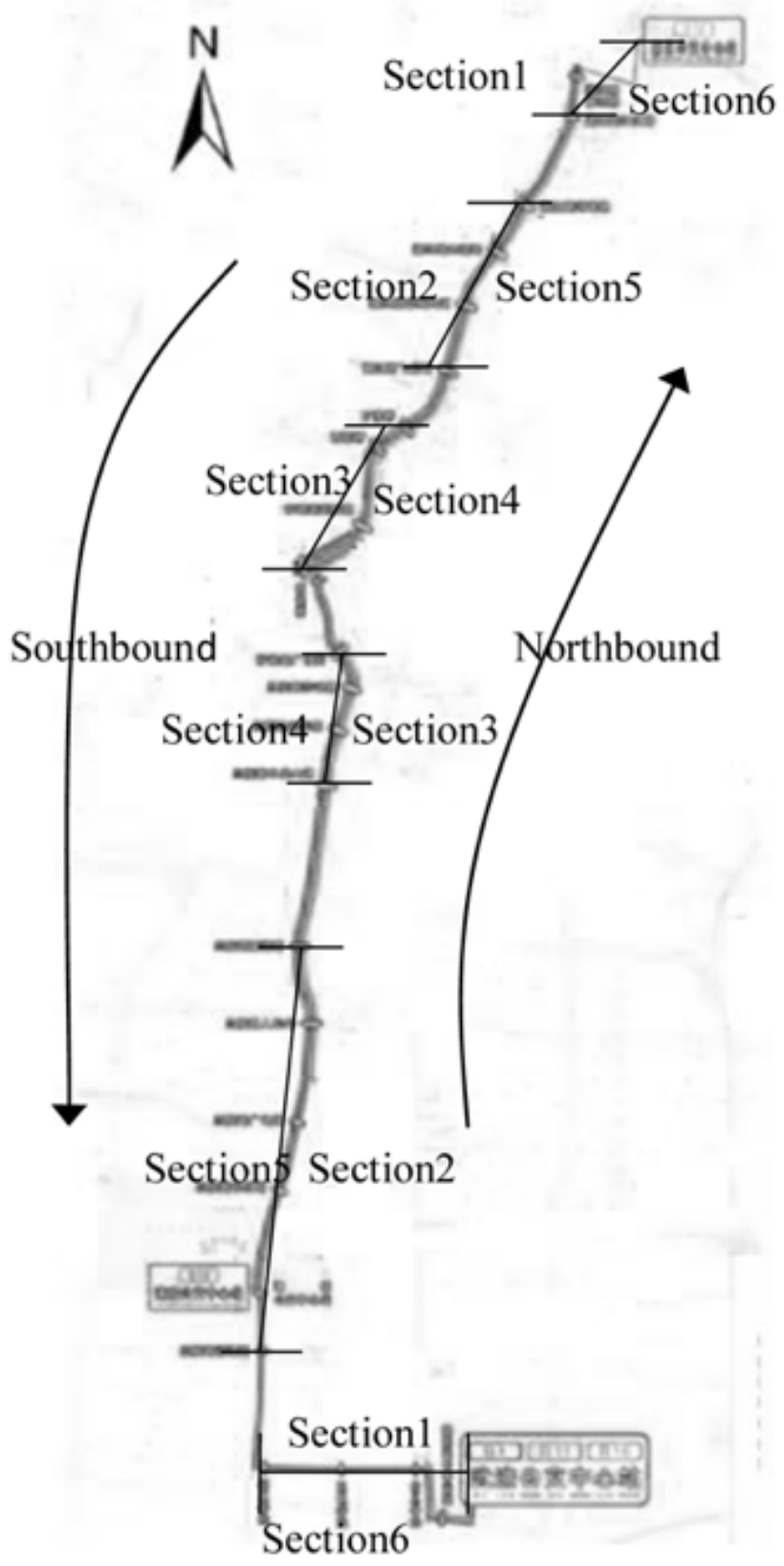

Figure 4. Section distribution for stops 

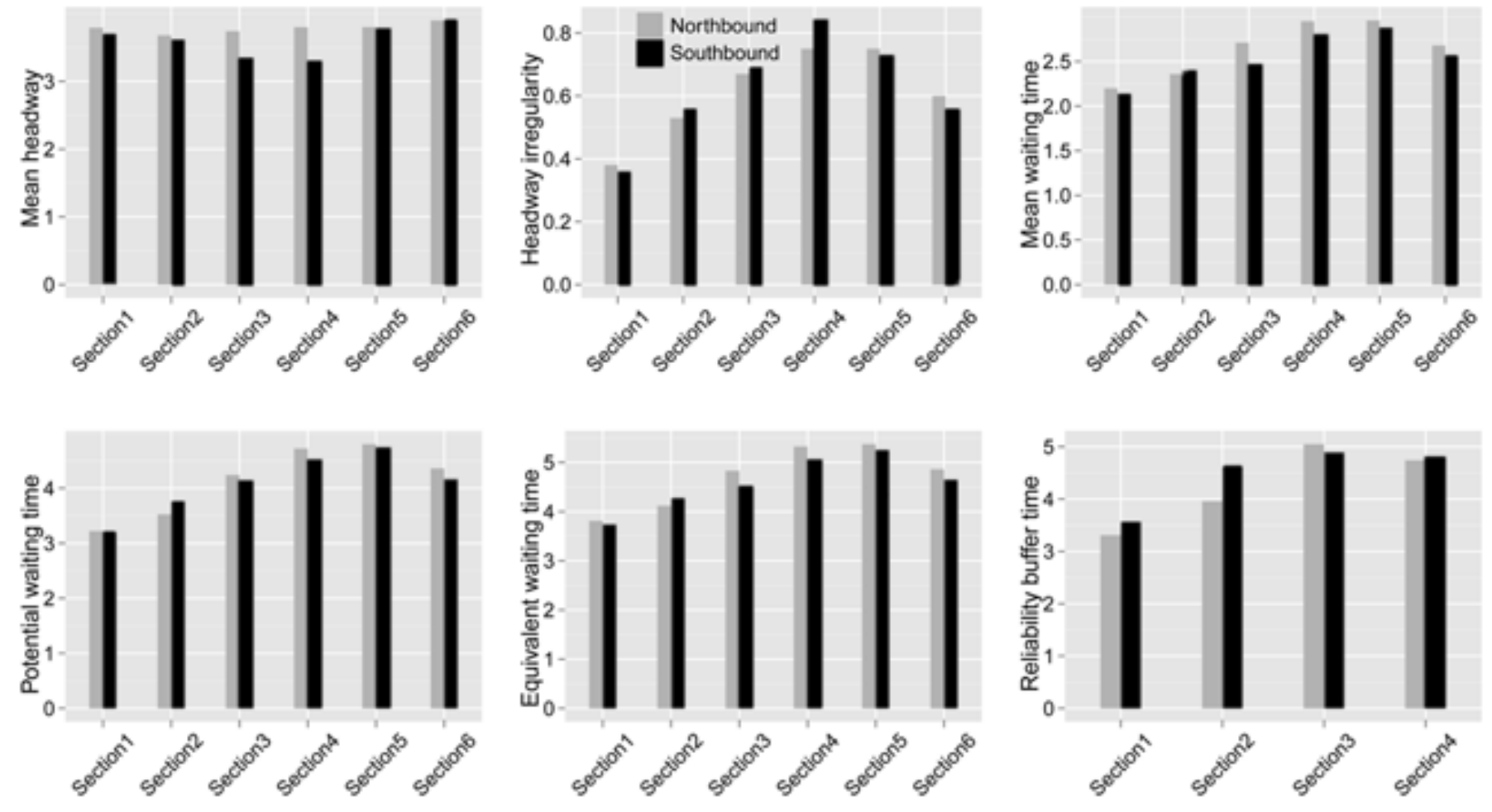

Figure $\mathbf{5}$. Service reliability by section 
Section 4 Northbound and Section3 Southbound in Figure 4 are the most congested sections. Service reliability should be the worst in these sections. Correspondingly, headway irregularity, mean waiting time, PWT, and EWT should be the highest. For Southbound, these four measures in Section 3 are not the highest; however, they are the highest in Section 4 and Section5. Therefore, it is possible that the effect of distance to the route's origin terminal on service reliability is stronger than the effect of the congestion level of areas where stops are located. Buses reaching Section 4 and Section 5 have traversed through the most congested section Southbound, and congestion may be propagated down, so this conclusion needs to be confirmed in future studies.

To analyze RBT by sections, OD pair3-OD pair6 in Figure 1 were selected. They are named Section 1 to Section4; the first OD pair closest to the route's origin terminal is Section 1 for Northbound and Southbound, as shown as Figure 6. RBT from Section1-Section4 for Northbound and Southbound are shown in Figure 5. RBT in Section1 is the lowest, reaches the highest in Section3, then begins to decline. In other words, RBT for OD pairs near the route's origin terminal is the lowest, gradually increases as the distance to the route's origin terminal increases, and becomes lower for OD pairs near the route's end, which also can be attributed to no schedule control for each stop.

Taking the variation trends of headway irregularity, mean waiting time, PWT, EWT, and RBT by sections into consideration, it appears that service reliability from both the operator and passenger perspectives is the best near the route's origin terminal, gradually deteriorates along the route, then improves when approaching the route's end.

\section{Comparison between Measures}

The ratio of PWT to mean waiting time is 1.5 to 1.71. In other words, passengers, on average, need to budget an extra 1.6 times mean waiting time to guarantee ontime arrival at their destinations with $95 \%$ probability. Passengers have experienced unreliable conventional bus service in China. When they use BRT, they budget a lot of extra time, which is longer than the actual waiting time. Changzhou BRT had operated only for 20 months as of August 2009. This phenomenon may change with the popularization of BRT.

The ratio of PWT at its origin to RBT for each OD pair is between 0.8 and 1.0. PWT accounts for more than 80 percent of RBT and indicates that passengers budget extra time beyond typical their journey time for the entire journey to arrive at their 


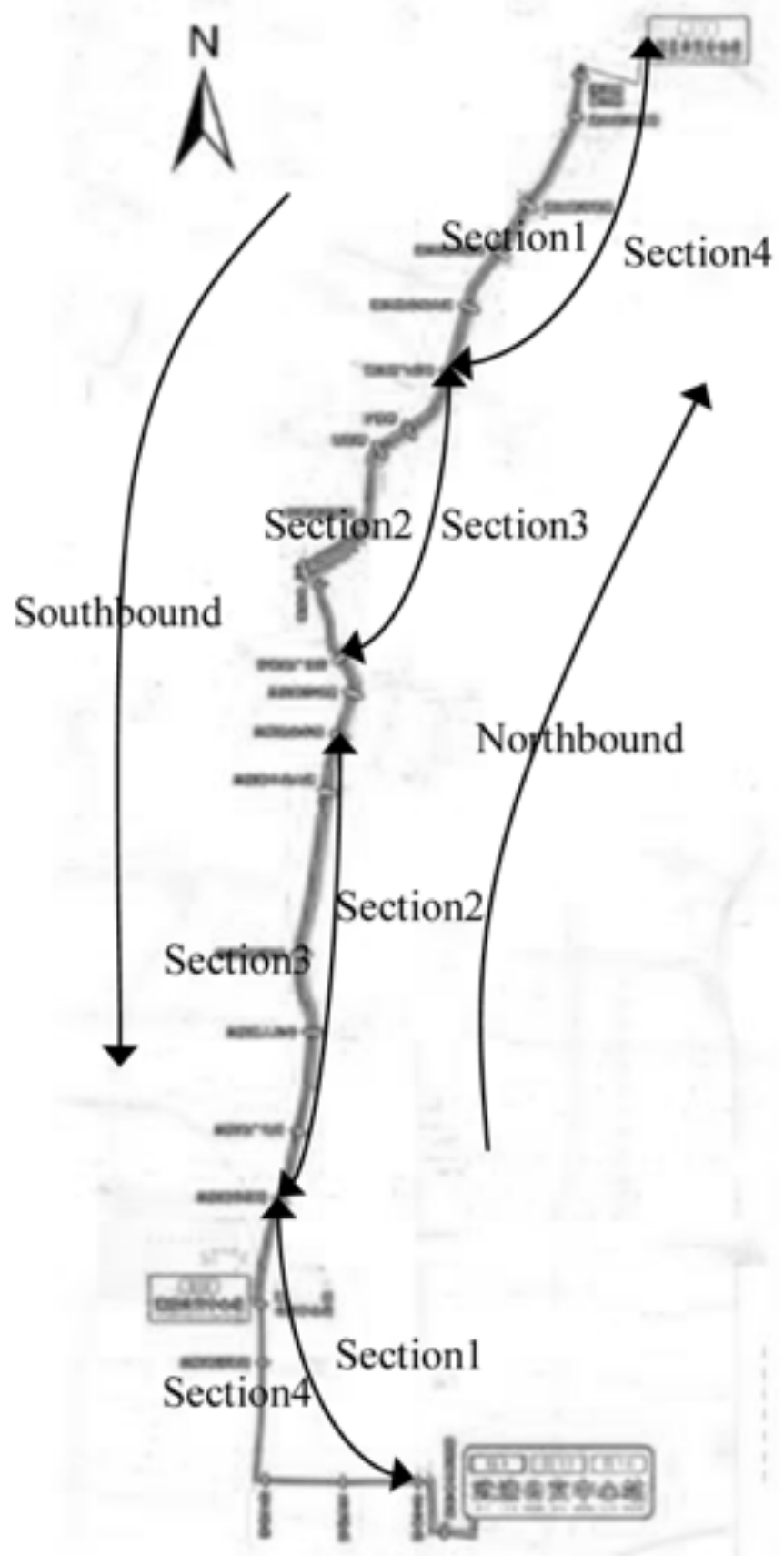

Figure 6. Sections distribution for OD pairs 
destinations at a high probability, primarily to ensure boarding the target bus. They believe in-vehicle travel time has low variation and, thus, they budget only small proportion for it. In other words, the extra time budgeted for bus waiting beyond mean waiting time contributes to more than 80 percent of the extra time budgeted for the entire journey, while only 20 percent of it is budgeted for in-vehicle travel time.

Passengers possibly rarely consider travel distance when deciding the extra time budgeted for an entire journey because no special relationship was found between travel distance and RBT. For example, the distance of OD pair2 is the longest in six OD pairs; its RBT Northbound is the highest, but second lowest Southbound. This conclusion needs to be further confirmed in future studies. To some extent, it can be implied from the conclusion that only 20 percent of the extra time budgeted for the entire journey is budgeted for in-vehicle travel time.

\section{Conclusions}

The service reliability of BRT was analyzed taking Changzhou BRT as an example, including value ranges of measures, temporal and spatial distributions, and comparisons. This framework can be applied to other BRT systems. Findings on the service reliability of Changzhou BRT are summarized below, and for each improvement, measures for the Changzhou transit agency are suggested.

Mean headway and mean waiting times for the Changzhou BRT are low, at 3.123.96 minutes and 2.17-2.82 minutes, respectively. Transit passengers often budget extra time for bus waiting and for the entire journey beyond typical waiting and journey times to ensure arrival at their destinations on time at a high probability. Passengers of Changzhou BRT need to budget an extra 3-5 minutes for their journey. The extra time budgeted for bus waiting contributes to more than 80 percent of the extra time budgeted for the entire journey, while only 20 percent is budgeted for in-vehicle travel time. Measures to reduce potential waiting time should be taken by the Changzhou transit agency, such as enhancing stop accessibility and educating passengers to board in an orderly manner. Headways of BRT are not as regular as expected; headway irregularity is between 0.34-0.79.

Service reliability of BRT varies from the Early to Evening periods. From the perspective of operators (i.e., headway irregularity), service reliability is the worst during the AM and PM peaks. However, from the perspective of passengers based on PWT and RBT, service reliability is the worst during the Early period followed by the AM Peak. Sometimes service reliability is different from the operator and pas- 
senger perspectives, so transit agencies should use multi-perspective measures to comprehensively evaluate service reliability. Specifically for the AM and PM peaks, headway irregularity is the highest and mean waiting time is the lowest, which are related to high service frequencies during these two periods. The Changzhou transit agency should implement some special traffic control treatments during peak hours, such as police guidance of traffic.

The spatial distribution of service reliability was analyzed by direction and section. For the direction dimension, service reliability is better To Downtown than it is From Downtown from both the operator and passenger perspectives. The Changzhou transit agency can improve service reliability through increasing service frequency From Downtown. For the section dimensions, service reliability from the operator and passenger perspectives is the same and is the best near the route's origin terminal, gradually deteriorating along the route, then improving when approaching the route's end. This can be partly attributed to no schedule control for each stop in China's bus service. The Changzhou transit agency should establish a schedule for each stop to improve en-route service reliability. Other improvement measures, such as using transit signal priority technology, dividing long routes into sub-routes, avoiding departure delays, and balancing passenger at bus doors, also can improve en-route service reliability.

\section{Acknowledgment}

This study is sponsored by National Key Basic Research Program of China (973 Program) (NO.2012CB725402) and National Nature Science Foundation of China (NO.51208099, NO.51108080 and NO.51308115). The authors thank Prof. Xuewu Chen in Southeast University for providing data and Zhan Zhao, Allison Lung, and Tracy Chen for proofreading this paper.

\section{Reference}

Abkowitz, M., L. Englisher, H. Slawin, R. Waksman, and N. Wilson. 1978. Transit service reliability. Report UMTA-MA-06-0049-78-1, United States Department of Transportation.

Cham, L. C. 2006. Understanding bus service reliability: A practical framework using AVL/APC data. Master's thesis, Department of Civil and Environmental Engineering, Massachusetts Institute of Technology. 
Chan, J. 2007. Rail transit OD matrix estimation and journey time reliability metrics using automated fare data. Master's thesis, Department of Civil and Environmental Engineering, Massachusetts Institute of Technology.

Cramer, A., J. Cucarese, M. Tran, A. Lu, and A. Reddy. 2008. Performance measurements on mass transit - New York City Transit Authority case study. Annual Meeting of the Transportation Research Board.

Deng, T. T., and J. D. Nelson. 2011. Recent developments in bus rapid transit: A review of the literature. Transport Reviews 31(1): 69-96.

Ehrlich, J. E. 2010. Application of automatic vehicle location systems towards improving service reliability and operations planning in London. Master's thesis, Department of Civil and Environmental Engineering, Massachusetts Institute of Technology.

Fjellstrom, K. 2010. Bus rapid transit in China. Built Environment 36(3): 363-374.

Furth, P. G., B. Hemily, T. H. J. Muller, and J. G. Strathman. 2006. Using archived AVLAPC data to improve transit performance and management. TCRP Report 113.

Furth, P. G., and T. H. J. Muller. 2006. Service reliability and hidden waiting time: Insights from automatic vehicle location data. Transportation Research Record 1955: 79-87.

Kittelson \& Associates, Inc., KFH Group, Inc., Parsons Brinckerhoff Quade \& Douglass, Inc., and Katherine Hunter-Zaworski. 2003. Transit Capacity and Quality of Service Manual, 2nd Edition. TCRP Report 100.

Levinson, H. S., S. Zimmerman, J. Clinger, J. Gast, S. Rutherford, and E. Bruhn. 2003. Bus Rapid Transit, Volume 2: Implementation Guidelines. TCRP Report 90.

Mo, Y. K. 2007. Study on optimization algorithm of route design of BRT. Traffic \& Transportation (1).

Osuna, E. E., and G. F. Newell. 1972. Control strategies for an idealized public transportation system. Transportation Science 6: 52-72.

Perk, V., M. Mugharbel, and M. Catalá. 2010. Impacts of bus rapid transit stations on surrounding single-family home values. Transportation Research Record 2144: 72-79.

Ryus, P. 2003. A summary of TCRP Report 88: A Guidebook for Developing a Transit Performance-Measurement System. TCRP project G-06. 
Uniman, D. L. 2009. Service reliability measurement framework using smart card data: Application to the London Underground. Master's thesis, Department of Civil and Environmental Engineering and Department of Urban Studies and Planning, Massachusetts Institute of Technology.

Xu, B. 2007. Design of urban road transit (BRT) System. Urban Roads Bridges \& Flood Control (5): 36-40.

\section{About the Authors}

YUEYING Huo (huoyueying2008@gmail.com) is an Assistant Professor at the Transportation Institute of Inner Mongolia University. She completed her Ph.D. at the School of Transportation in Southeast University and studied at the University of British Columbia. Her research interests include public transit planning, capacity, and service quality of public transit.

JINHUA ZHAO (jinhua@mit.edu) is an Assistant Professor in Department of Urban Studies and Planning in Massachusetts Institute of Technology. He holds a Ph.D. in City and Regional Planning and a master's degree in Transportation and City Planning from the Massachusetts Institute of Technology. His research interests include urban development and planning in China, urban transportation systems in China, transportation economics, public transportation management, and urban information systems.

WeNQUAN LI (wenqli@seu.edu.cn) is a Professor at the School of Transportation at Southeast University. He holds a Ph.D. from the School of Transportation at Southwest Jiaotong University. His research interests include transportation planning and management, traffic flow theory, traffic safety, and public transit.

XIAOJIAN Hu (huxiaojian@seu.edu.cn) is an Assistant Professor at the School of Transportation in Southeast University, at which he completed his Ph.D. His research interests include transportation management, traffic flow theory, and traffic control. 